\title{
AUTOMATIC TUNING OF PROPORTIONAL- INTEGRAL-DERIVATIVE (PID) CONTROLLER USING PARTICLE SWARM OPTIMIZATION (PSO) ALGORITHM
}

\author{
S. J. Bassi ${ }^{1}$, M. K. Mishra ${ }^{2}$ and E. E. Omizegba ${ }^{3}$ \\ ${ }^{1}$ Department of Computer Engineering, University of Maiduguri, Borno State, Nigeria \\ sjbassi 74 @yahoo.co.uk \\ ${ }^{2}$ Department of Computer Engineering, University of Maiduguri, Borno State, Nigeria \\ mishrasoftlegmail.com \\ ${ }^{3}$ Electrical and Electronics Engineering Programme, Abubakar Tafawa Balewa \\ University, \\ P.M.B 0248, Bauchi, Bauchi State, Nigeria \\ omizegbaee@yahoo.co.uk
}

\begin{abstract}
The proportional-integral-derivative (PID) controllers are the most popular controllers used in industry because of their remarkable effectiveness, simplicity of implementation and broad applicability. However, manual tuning of these controllers is time consuming, tedious and generally lead to poor performance. This tuning which is application specific also deteriorates with time as a result of plant parameter changes. This paper presents an artificial intelligence (AI) method of particle swarm optimization (PSO) algorithm for tuning the optimal proportional-integral derivative (PID) controller parameters for industrial processes. This approach has superior features, including easy implementation, stable convergence characteristic and good computational efficiency over the conventional methods. Ziegler-Nichols, tuning method was applied in the PID tuning and results were compared with the PSO-Based PID for optimum control. Simulation results are presented to show that the PSO-Based optimized PID controller is capable of providing an improved closed-loop performance over the Ziegler-Nichols tuned PID controller Parameters. Compared to the heuristic PID tuning method of Ziegler-Nichols, the proposed method was more efficient in improving the step response characteristics such as, reducing the steady-states error; rise time, settling time and maximum overshoot in speed control of DC motor.
\end{abstract}

\section{KEYWORDS:}

PID Controller, Particle swarm optimization algorithm, Ziegler- Nichols method, Simulation 


\subsection{INTRODUCTION}

The PID controller is regarded as the workhorse of the process industry. Today, many industrial processes are controlled using proportional-integral-derivative (PID) controllers. The popularity of the PID controllers can be attributed to their good performance in a wide range of operating conditions, functional simplicity, which allows Engineers to operate them in a simple, straightforward manner and familiarity, with which it is perceived amongst researchers and practitioners within the process control industries (Pillay and Govender, 2007). In spite of its widespread use, one of its main short-comings is that there is no efficient tuning method for this type of controller (Åström and Hägglund, 1995).

Several methods have been proposed for the tuning of PID controllers. Among the conventional PID tuning methods, the Ziegler-Nichols method (Ogata, 1987) may be the most well known technique. For a wide range of practical processes, this tuning approach works quite well. However, sometimes it does not provide good tuning and tends to produce a big overshoot. Therefore, this method usually needs retuning before applied to control industrial processes. To enhance the capabilities of traditional PID parameter tuning techniques, several intelligent approaches have been suggested to improve the PID tuning, such as those using genetic algorithms (GA) (Mahony, et al, 2000; Wang and Tracht, 2003; Krishnakumar and Goldberg, 1992; Varsek, et al, 1993) and the particle swarm optimization (PSO) (Gaing, 2004; Solihin, et al 2011). With the advance of computational methods in the recent times, optimization algorithms are often proposed to tune the control parameters in order to find an optimal performance (Gaing, 2004; Solihin, et al 2011).

It has been asserted that more than half of the industrial controllers in use today utilize PID or modified PID control schemes (Ogata, 2005). This wide spread acceptance of the PID controllers is largely attributed to their simplicity and robust performance in wide range of operating conditions. One major problem faced in the deployment of PID controllers is the proper tuning of gain values (Visioli, 2001). Over the years, various heuristic techniques were proposed for tuning the PID controller. Among the earliest methods is the classical Ziegler-Nichols tuning procedure, however, it is difficult to determine optimal or near optimal parameters with this because most industrial plants are often very complex having high order, time delays and nonlinearities (Kwok et al., 1993, Gaing, 2004, and Krohling and Rey, 2001).

This paper attempts to develop an artificial intelligence(AI) automatic PID tuning scheme using PSO algorithm that can automatically acquire (or re-adapt) the PID parameters during plant operation in a routine way. The result is expected to show the effectiveness of the modern optimization such as PSO in control engineering applications. PSO algorithm is a stochastic algorithm based on principles of natural selection and search algorithm. There, are many evidences of intelligence for the posed domains in animals, plants, and generally living systems. For example, ants foraging, birds flocking, fish schooling, bacterial chemotaxis are some of the well-known examples in category.

\subsection{PID CONTROLLER TUNING METHODS}

The goal of tuning the PID controller is to determine parameters that meet closed loop system performance specifications, and to improve the robust performance of the control loop over a wide range of operating conditions. Practically, it is often difficult to simultaneously achieve all 
International Journal of Artificial Intelligence \& Applications (IJAIA), Vol.2, No.4, October 2011

of these desirable qualities. For example, if the PID controller is adjusted to provide better transient response to set point change, it usually results in a sluggish response when under disturbance conditions. On the other hand, if the control system is made robust to disturbance by choosing conservative values for the PID controller, it may result in a slow closed loop response to a set point change. A number of tuning techniques that take into consideration the nature of the dynamics present within a process control loop have been proposed (see Ziegler and Nichols, 1942; Cohen and Coon, 1953; Åström and Hägglund, 1984; De Paor and O'Malley, 1989; Zhuang and Atherton, 1993; Venkatashankar and Chidambaram, 1994; Poulin and Pomerleau, 1996; Huang and Chen, 1996). All these methods are based upon the dynamical behavior of the system under either open-loop or closed-loop conditions.

\subsection{PROBLEM FORMULATION}

PID controller consists of Proportional, Integral and Derivative gains. The feedback control system is illustrated in Fig. 1 where $r, e, \mathrm{u}, y$ are respectively the reference, error, controller output and controlled variables.

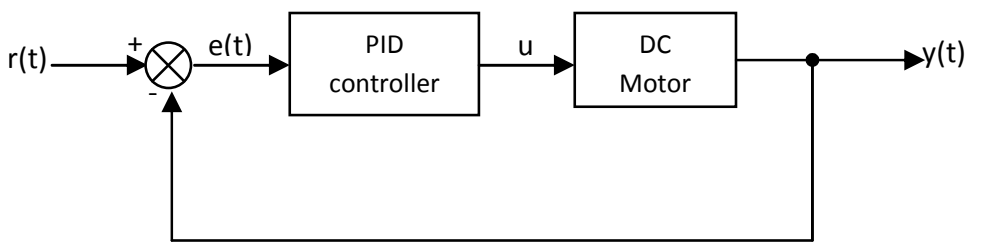

Figure 1: A common feedback control system

The PID controller is described in equation (1) as:

$$
u(t)=K_{p} e(t)+K_{i} \int_{0}^{t} e(t) d t+K_{d} \frac{d e}{d t}
$$

Where $u_{t}$ is the controller output, $e_{t}$ is the error, and $t$ is the sampling instance. The factors $k_{p}, k_{i}$ and $k_{d}$ are the proportional, integral and derivatives gains (or parameters) respectively that are to be tuned. The DC motor model is described in equation (2) as:

$$
G_{c}(s)=\frac{6.3223 s^{2}+18 s+12.812}{s}
$$

Furthermore, performance index is defined as a quantitative measure to depict the system performance of the designed PID controller. Using this technique an 'optimum system' can often be designed and a set of PID parameters in the system can be adjusted to meet the required specification. For a PID- controlled system, there are often four indices to depict the system performance: ISE, IAE, ITAE and ITSE. Therefore, for the PSO-based PID tuning, the ITAE performance index given in equation (3) will be used as the objective function. In other word, the objective in the PSO-based optimization is to seek a set of PID parameters such that the feedback control system has minimum performance index. 


$$
\text { ITAE }=\int_{0}^{\infty} t \mid e(t) d t
$$

\subsection{TUNING OF PID CONTROLLER USING ZIEGLER NICHOLS METHOD}

The first method of Z-N tuning is based on the open-loop step response of the system. The openloop system's S shaped response is characterized by the parameters, namely the process time constant $\mathrm{T}$ and $\mathrm{L}$. These parameters are used to determine the controller's tuning parameters. The second method of Z-N tuning is closed-loop tuning method that requires the determination of the ultimate gain and ultimate period. The method can be interpreted as a technique of positioning one point on the Nyquist curve (Astrom and Hagglund, 1995). This can be achieved by adjusting the controller gain $(\mathrm{Ku})$ till the system undergoes sustained oscillations (at the ultimate gain or critical gain), whilst maintaining the integral time constant (Ti) at infinity and the derivative time constant (Td) at zero. This paper uses the second method as shown in table 1.

Table 1: Ziegler-Nichols open-loop tuning rule

\begin{tabular}{llll}
\hline \hline Controller & $K_{P}$ & $T_{I}$ & $T_{D}$ \\
\hline $\mathrm{P}$ & $\frac{T_{p}}{L_{p} K_{p}}$ & $\infty$ & 0 \\
& $0.9 \frac{T_{p}}{L_{p} K_{p}}$ & $3.33 L_{p}$ & 0 \\
\hline $\mathrm{PI}$ & $1.2 \frac{T_{p}}{L_{p} K_{p}}$ & $2 L_{p}$ & $0.5 L_{p}$ \\
\hline PID & & \\
\hline \hline
\end{tabular}

\subsection{OVERVIEW OF PARTICLE SWARM OPTIMIZATION (PSO) ALGORITHM}

PSO is optimization algorithm based on evolutionary computation technique. The basic PSO algorithm is developed from research on swarm such as fish schooling and bird flocking (Ou, \& Lin,2006). After it was firstly introduced in 1995 (Kennedy \& Eberhart, 1995), a modified PSO was then introduced in 1998 to improve the performance of the original PSO algorithm. A new parameter called inertia weight is added (Shi \& Eberhart, 1998). This is a commonly used PSO 
International Journal of Artificial Intelligence \& Applications (IJAIA), Vol.2, No.4, October 2011

algorithm where inertia weight is linearly decreasing during iteration in addition to another common type of PSO algorithm which is reported by Clerc (Eberhart, \& Shi, 2000). The later is the one used in this paper.

In PSO, instead of using genetic operators, individuals called as particles are "evolved" by cooperation and competition among themselves through generations. A particle represents a potential solution to a problem. Each particle adjusts its flying according to its own flying experience and its companion flying experience. Each particle is treated as a point in a Ddimensional space. The $i$ th particle is represented as $X_{I}=\left(X_{i !}, X_{i 2}, \ldots, X_{i D}\right)$. The best previous position (giving the minimum fitness value) of any particle is recorded and represented as $P_{I}=\left(P_{i !}, P_{i 2}, \ldots, P_{i D}\right)$, this is called pbest. The index of the best particle among all particles in the population is represented by the symbol $g$, called as gbest. The velocity for the particle $i$, is represented as $V_{I}=\left(V_{i !}, V_{i 2}, \ldots, V_{i D}\right)$. The particles are updated according to equations (4) and (5).

$$
\begin{aligned}
& v_{i, m}^{(t+1)}=w \cdot v_{i, m}^{(t)}+c_{1} * \operatorname{rand}() *\left(\text { pbest }_{i, m}-x_{i, m}^{(t)}+c_{2} * \operatorname{rand}() *\left(\text { gbest }_{m}-x_{i, m}^{(t)}\right)\right. \\
& x_{i, m}^{(t+1)}=x_{i, m}^{(t)}+v_{i, m}^{(t+1)}
\end{aligned}
$$

Where, $\mathrm{c} 1$ and $\mathrm{c} 2$ are two positive constant. While rand () is random function between 0 and 1 , and $n$ represents iteration. Equation (4) is used to calculate particle's new velocity according to its previous velocity and the distances of its current position from its own best experience (position) and the group's best experience. Then the particle flies toward a new position according to Equation (5). The performance of each particle is measured according to a pre-defined fitness function (performance index), which is related to the problem to be solved. Inertia weight, $w$ is brought into the equation to balance between the global search and local search capability (Shi \& Eberhart, 1998). It can be a positive constant or even positive linear or nonlinear function of time. It has been also shown that PSO with different number of particles (swarm size) has reasonably similar performance (Shi, \& Eberhart, 2001)

\subsection{IMPLEMENTATION OF PSO-BASED PID TUNING}

Stochastic Algorithm can be applied to the tuning of PID controller gains to ensure optimal control performance at nominal operating conditions. PSO algorithm is employed to tune PID gains/parameters (Kp, Ki, Kd) using the model in Equation (2). PSO algorithm firstly produces initial swarm of particles in search space represented by matrix. Each particle represents a candidate solution for PID parameters where their values are set in the range of 0 to 100 . For this 3-dimentional problem, position and velocity are represented by matrices with dimension of $3 x$ Swarm size. The swarm size is the number of particle where 100 are considered a lot enough. A good set of PID controller parameters can yield a good system response and result in minimization of performance index in Equation (3). 
International Journal of Artificial Intelligence \& Applications (IJAIA), Vol.2, No.4, October 2011

\subsection{SIMULATION RESULTS}

Implementing control design on any physical system involves some leap of confidence. In this setting, we have attempted to design a controller for a dynamic model of DC motor. A standard test model as considered is taken for stability study of DC motor with PSO-PID tuning controller. The test model in Fig. 1 shows the block diagram of the control system.

In the conventionally Z-N tuned PID controller, the plant response produces high overshoot and long settling time, but a better performance obtained with the implementation of PSO-based PID controller tuning. These are shown in Table 2. Furthermore, Fig. 2 shows the curve of the PID parameters during optimization to see the convergence of the performance index optimized solution. The PID parameters are obtained for 100 iterations.

Table 2: Optimized PID Parameters

\begin{tabular}{l|c|c|c}
\hline Tuning Method & $\mathrm{K}_{\mathrm{p}}$ & $\mathrm{K}_{\mathrm{i}}$ & $\mathrm{K}_{\mathrm{d}}$ \\
\hline Z-N PID & 30.318 & 39.42 & 12.812 \\
\hline $\begin{array}{l}\text { PSO-PID } \\
\text { (ITAE) }\end{array}$ & 22.8070 & 2.0734 & 17.4628 \\
\hline \hline
\end{tabular}

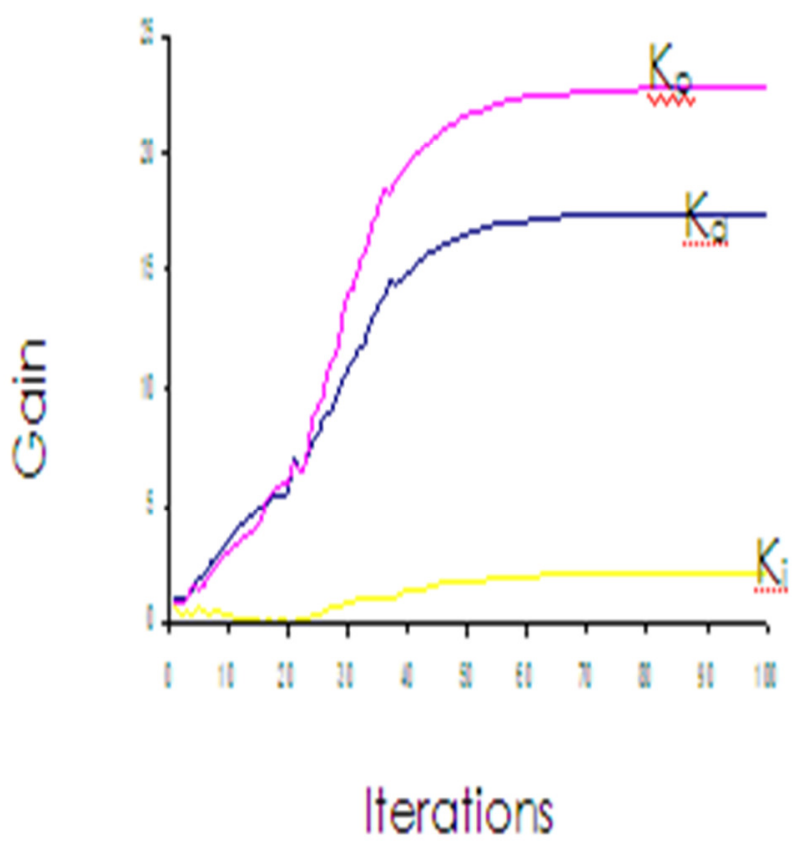




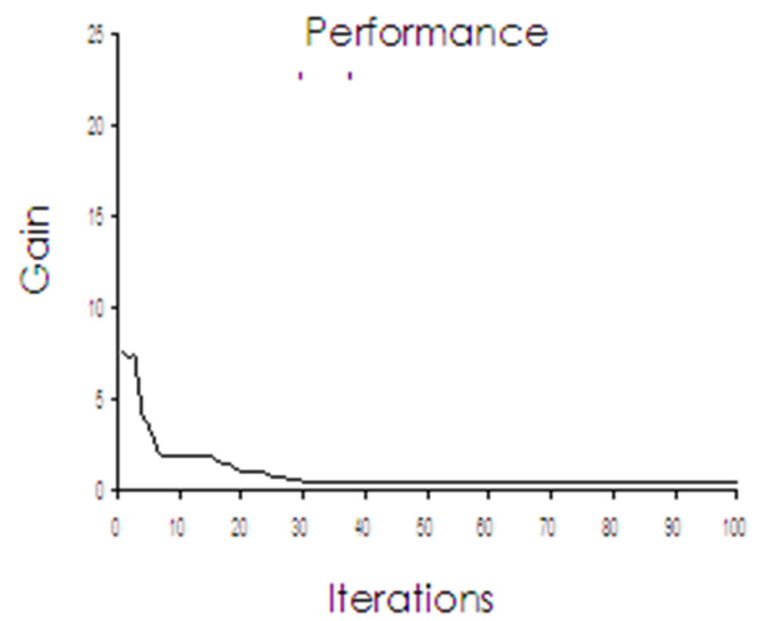

Figure 2: The parameters and the performance index trajectory (PSO-PID)

Comparative results for the PID controllers are given in Table 3 where the step response performance is evaluated based on the rise time, settling time and overshoot. The corresponding plot for the step responses are shown in Fig. 3. Finally, this result is only preliminary research. To further investigate the effectiveness of the proposed method, some work may be done such as:

- Comparison of the PSO-PID with other artificial intelligence (AI) optimization techniques, like Genetic Algorithm (GA).

- Instead of PSO algorithm, others optimizer such as Differential Optimization can be used.

- Different objective functions other than ITAE performance index that is already used.

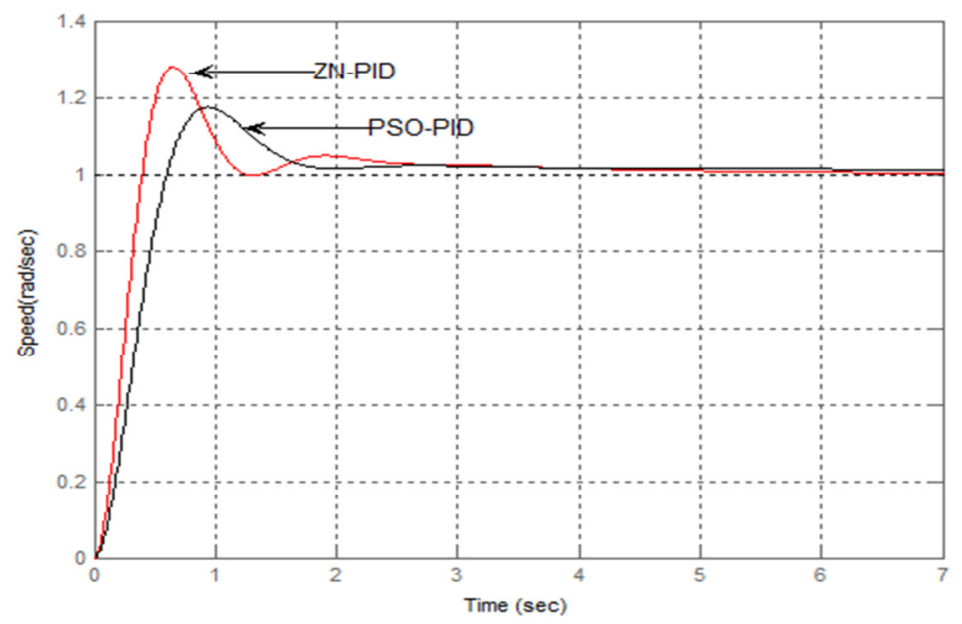

Figure 3: Comparison of the step response for PID controllers 
Table 3: Comparison of ZN-PID and PSO-PID for Brushless DC Motor

\begin{tabular}{c|c|c|c|c|c|c}
\hline \hline METHOD & $\begin{array}{c}\text { Rise time } \\
(\mathbf{s})\end{array}$ & $\begin{array}{c}\text { Settling } \\
\text { time (s) }\end{array}$ & Overshoot (\%) & P & I & D \\
\hline Z-N & 0.307 & 3.44 & 28.1 & 30.3 & 39.4 & 12.8 \\
\hline PSO & 0.418 & 3.17 & 17.4 & 22.8 & 2.1 & 17.5 \\
\hline \hline
\end{tabular}

\subsection{CONCLUSION}

PID controllers are a widespread control solution due to their simple architecture, generally acceptable control performance and ease of use. Unlike other control options, PID controllers do not require the user to have an extensive background in mathematics, control theory or electrical engineering to understand them. They are found in a wide variety of applications, and if properly tuned will outperform almost any other control option. It is in tuning the controllers that the greatest gains in performance may be found. A wide variety of tuning methods exist, although of the two discussed in this article, the PSO algorithm method provides the most effective approach to tuning a controller.

It was shown, by comparison of their responses that the PSO algorithm has outperformed the Ziegler Nichols method in terms of the system overshoot, settling time and rise time. Also the performance index value obtained with the proposed PSO algorithm is less than of the ZieglerNichols method. The performance of the PSO algorithm method of tuning a PID controller has been proved to be better than the heuristic Ziegler-Nichols method.

From the results, the designed PID controller using PSO algorithm shows superior performance over the traditional method of Ziegler-Nichols, in terms of the system overshoot, settling time and rise time. However, the traditional method provides us with the initial PID gain values for optimal tuning. Therefore the benefit of using a modern artificial intelligence optimization approach is observed as a complement solution to improve the performance of the PID controller designed by conventional method. Of course there are many techniques can be used as the optimization tools and PSO is one of the recent and efficient optimization tools.

\section{REFERENCES}

[1] A.Varsek, T. Urbacic and B. Filipic, 1993, Genetic Algorithms in Controller Design and Tuning, IEEE Trans. Sys. Man and Cyber, Vol. 23/5, pp1330-1339.

[2] Astrom, K. J. and T., Hagglund, 1995, PID Controllers: Theory, Design and Tuning, ISA, Research Triangle, Par, NC. 
International Journal of Artificial Intelligence \& Applications (IJAIA), Vol.2, No.4, October 2011

[3] B.Nagaraj,S.Suba and B.Rampriya: Tuning Algorithms for PID Controller Using Soft Computing Techniques; International Journal of Computer Science and Network Security(IJCSNS), VOL.8 No.4, April 2008. pp278-281.

[4] Clerc, M. ,1999, The Swarm and the queen: towards a deterministic and adaptive particle swarm optimization. Proceedings of the Conference on Evolutionary Computation, pp. 1951-1957.

[5] Cohen G.H. and Coon G.A., "Theoretical consideration of retarded control", Trans. ASME, Vol. 75, pp. 827-834, 1953

[6] Eberhart, R.C. and Shi, Y.H., 2000, Comparing inertia weights and constriction factors in particle swarm optimization. Proc. The 2000 Congress on Evolutionary Computation. Vol.1, pp. 84-88.

[7] Engineering and Information Technology 2011

[8] Gaing, Z.L., 2004, A particle swarm optimization approach for optimum design of PID controller in AVR system. IEEE Transaction on Energy Conversion, Vol.19(2), pp.384-391.

[9] Hugo, J. Alan, 2002, Process Controller Performance Monitoring and Assessment, http/www.controlartsinc.com/support/articles/PerformanceAssessment.PDF. Accessed 31/6/10

[10] K Ogata, 1987, Modern Control Systems, University of Minnesota, Prentice Hall.

[11] K Ogata, 2005, Modern Engineering (fifth edition), University of Minnesota, Prentice Hall.

[12] K. Krishnakumar and D. E. Goldberg, 1992, Control System Optimization Using Genetic Algorithms, Journal of Guidance, Control and Dynamics, Vol. 15, No. 3, pp. 735-740.

[13] Kennedy, J. and Eberhart, R.C., 1995, Particle swarm optimization. Proc. IEEE International Conference on Neural Networks (Perth,Australia), IEEE Service Center, Piscataway, NJ, pp. IV: 1942- 1948.

[14] Krohling RA, Rey JP., 2001, Design of optimal disturbance rejection PID controllers using genetic algorithm. IEEE Trans Evol Comput;5: pp. 78-82.

[15] Kwok,D.P.,T.P.Leung and F.Sheng, 1993, Genetic algorithm for optimal dynamic control of robot arms. Proceedings of the International Conference on Industrial Electronics, Control and Instrumentation.15-19 November,San Francisco,CA, pp. 380-385.

[16] Mahmud Iwan Solihin, Lee Fook Tack and Moey Leap Kean, Tuning of PID Controller Using Particle Swarm Optimization (PSO), Proceeding of the International Conference on Advanced Science,

[17] Mitsukura Y, Yamamoto T, Kaneda M., June 1999, A design of self-tuning PID controllers using a genetic algorithm. In: Proc Am Contr Conf, San Diego, CA, pp. 1361-5.

[18] Nagaraj B, Subba S and Rampriya B, 2005, Tuning Algorithm for PID Using Soft-Computing Techniques. International Journal of Computer Science and Network Security, Vol 8, No 4 April ,pp. $278-289$.

[19] Ou, C. and Lin, W., 2006, Comparison between PSO and GA for parameters optimization of PID controller. Proc. IEEE International Conference on Mechatronics and Automation. Luoyang, China. 
International Journal of Artificial Intelligence \& Applications (IJAIA), Vol.2, No.4, October 2011

[20] Pillay N. and Govender P., 2007, A Particle Swarm Optimization Approach for Model Independent Tuning of PID Control Loop, IEEE Africon 2007, IEEE Catalog: 04CH37590C, ISBN: 0-7803-8606-X.

[21] Q.Wang, P Spronck and R Tracht, 2003, An Overview of Genetic Algorithms Applied to Control Engineering Problems. Proceedings of the Second International Conference on Machine Learning and Cybernetics.

[22] Qu Sun, Renhou Li and Ping and Zhang, 2003, Stable and Optimal Adaptive Fuzzy Control of Complex Systems using Fuzzy Dynamic Model. Fuzzy Sets and Systems, No 133 pp. 1 - 17.

[23] Rasmussen H., 2002, Automatic Tuning of PID Regulator. http://www.volgas.dk/nores/auto.pdf downloaded 30/6/10.

[24] Schei, Tor Steiner, 1994, Automatic Tuning of PID Controllers Based on Transfer Function Estimation, Automatica, pp. pp. $1983-1989$.

[25] Seng TL, Khalid MB, Yusof R., 1999, Tuning of a neuro-fuzzy controller by genetic algorithm. IEEE Trans Syst Man Cybern B,29, pp. 226-36.

[26] Shi, Y.H. and Eberhart, R.C., 1998, A modified particle swarm optimizer. IEEE International Conference on Evolutionary Computation, Anchorage, Alaska.

[27] Shi, Y.H. and Eberhart, R.C., 2001, Fuzzy Adaptive Particle Swarm Optimization. Proc. Congress on Evolutionary Computation. Seoul,Korea.

[28] T. O.Mahony, C J Downing and K Fatla, 2000, Genetic Algorithm for PID Parameter Optimization: Minimizing Error Criteria, Process Control and Instrumentation, University of Stracthclyde, pp. 148- 153.

[29] Visioli A., 2001, Tuning of PID controllers with fuzzy logic. Proc Inst Elect Eng Contr Theory Appl, 148(1), pp. 1-8.

[30] Zhong Jinghua ,2006, PID Controller Tuning: A Short Tutorial, http://saba.kntu.ac.ir/eecd/pd/download/PIDtutorial.pdf downloaded 1/7/2010 